\title{
UTILIZAÇÃO DE PLANEJAMENTO EXPERIMENTAL PARA DESENVOLVIMENTO DE NOVAS FORMULAÇÕES PARA LUBRIFICANTES DE ÓLEO DE MORINGA OLEIFERA LAM EPOXIDADO
}

\author{
M. S. SILVA ${ }^{1}$, D. K. da S. GUIMARÃES ${ }^{2}$, T. N. de CASTRO DANTAS ${ }^{3}$, A. A. DANTAS
} $\mathrm{NETO}^{1}$.

${ }^{1}$ Universidade Federal do Rio Grande do Norte, Programa de Pos Graduação em Engenharia Quimica - PPGEQ,

${ }^{2}$ Universidade Federal do Rio Grande do Norte, Programa de Pos Graduação em Engenharia de Petroleo - PPGEP,

${ }^{3}$ Universidade Federal do Rio Grande do Norte , Instituto de Química - PPGQ.

E- mail para contato: susana_mss@yahoo.com.br

RESUMO - O objetivo principal do trabalho foi obter um biolubrificante hidráulico a partir do óleo Moringa oleifera Lam epoxidado, cujas propriedades oxidativas, acidez e a instabilidade térmica do óleo vegetal in natura são melhoradas. Para o desenvolvimento desse trabalho, e com o intuito de melhorar as características físicoquímicas do epóxido foram feitas aditivações do óleo epoxidado com um antiespumante, desemulsificante e utilizando um planejamento fatorial $2^{3}$ com composto central variando o inibidor de corrosão (IC), extrema pressão (EP) e antioxidante (BHA), sendo posteriormente caracterizado. A análise da superfície de resposta do planejamento fatorial concluiu que para o melhor desempenho do fluido como lubrificante hidráulico é necessário $0,69 \mathrm{~mL}$ de IC, 3,9 g de EP e 0,39 g de BHA obtendo assim resultados de desempenho superior para o óleo epoxidado, onde, o índice de acidez total foi igual a $0,13 \mathrm{mg} \mathrm{KOH} / \mathrm{g}, 211^{\circ} \mathrm{C}$ de ponto de fulgor, $5 \mathrm{~h} 13 \mathrm{~min}$ de estabilidade oxidativa, $83,95 \mathrm{~mm}^{2} / \mathrm{s}$ de viscosidade e $1 \mathrm{~A}$ para o teste de corrosão ao cobre.

\section{INTRODUÇÃO}

Atualmente tem-se observado um significativo investimento em estudos cuja finalidade é a substituição de produtos provenientes da indústria petrolífera por derivados de fontes renováveis. O objetivo é minimizar os impactos causados ao meio ambiente desde o processo de exploração até a obtenção de derivados, como derramamentos, geração de efluentes, principalmente o dióxido de carbono, responsável pelo aumento do efeito estufa, ou até mesmo produção de resíduos não biodegradáveis (Ereda, 2004). 


\section{9 a 22 de outubro de 2014 \\ Florianópolis/SC}

Uma fonte alternativa que vem sendo bastante estudada é o uso de óleos vegetais para obtenção de lubrificantes. O estudo visando à substituição de lubrificantes convencionais por lubrificantes a base de óleo vegetal tem se tornado bastante atraente, pois estes apresentam características desejáveis, como boa lubricidade, não poluentes, não tóxicos, propriedades anticorrosivas, alto ponto de fulgor, apresentam alto índice de viscosidade e baixa volatilidade, além de serem mais baratos que os convencionais (Honary, 2001; Erhan et al., 2008). Porém, a presença de insaturações na molécula de ácidos graxos componentes do óleo vegetal conferem aos biolubrificantes uma baixa estabilidade térmica e oxidativa, além de baixo desempenho em baixas temperaturas (Perez, 2009; Arbain e Salimon, 2011; Salih et al., 2011).

A modificação da estrutura química do óleo vegetal, por diferentes rotas tem sido uma alternativa para obtenção de lubrificantes de base vegetal com alta estabilidade oxidativa. As principais rotas são a transesterificação e a epoxidação. Esta última se torna uma alternativa interessante devido por não necessitar de catalizador e utilizar baixas temperaturas para o processo de síntese do epóxido, tornando a operação mais viável economicamente. (Hwang et al., 2003).

O óleo de Moringa oleifera Lam apresenta elevado teor de ácidos graxos insaturados, sendo predominante o ácido oleico, apresenta elevado ponto de fulgor e uma maior resistividade à oxidação, seja a altas temperaturas ou em condições ambientes, garantindo a ele também uma boa estabilidade oxidativa (Abdulkarim, 2007).

O objetivo deste trabalho foi obter um novo biolubrificantes hidráulicos a partir do óleo de Moringa (Moringa oleifera Lam) epoxidado via ácido perfórmico, utilizando o planejamento experimental fatorial $2^{3}$ para o estudo dos efeitos dos pacotes dos aditivos nas propriedades e na estabilidade dos biolubrificantes.

\section{METODOLOGIA EXPERIMENTAL}

\subsection{Preparação e caracterização de lubrificante de base vegetal}

A base vegetal para formulação de lubrificantes foi preparada por reação de epoxidação, na qual a ligação dupla é convertida em anel oxirano, esta conversão visa melhorar a estabilidade térmica e oxidativa bem como a capacidade de lubrificação do óleo vegetal. O óleo moringa epoxidado foi preparado via ácido perfórmico gerado in situ. Esta reação foi realizada utilizando a razão molar de peróxido de hidrogênio/ácido fórmico/ligações duplas igual a 1:1:1,5, em um balão de $250 \mathrm{~mL}$ acoplado a um condensador de refluxo, sob aquecimento e com agitação constante $(500 \mathrm{rpm})$. Para evitar a hidrólise dos óleos vegetais, o peróxido de hidrogênio foi adicionado fracionadamente durante a primeira hora de reação.

O óleo epoxidado foi analisado utilizando metodologia das normas ASTM, a seguir: viscosidade a $40^{\circ} \mathrm{C}(\mathrm{ASTM}$ D 2983), ponto de fulgor (ASTM D-92/90), estabilidade oxidativa(ASTM D7525 e ASTM D7545), corrosão ao cobre (ASTM D- 1298/89), densidade a 
40( ASTM D- 1298/89), oxigênio oxirânico (IUPAC, 1987) e índice de iodo (AOCS Cd 125/90).

\subsection{Aditivos dos biolubrificantes}

Foram utilizados na aditivação dos biolubrificantes: o antiespumante (LIOFOAM 149), desemulsificante (LIOVAC OAS700), inibidor de corrosão - IC (LIOVAC 3355), extrema pressão - EP (LIOVAC 89ss) cedidos pela da Miracema-Nuodex Ltda e o antioxidante 2-tertbutyl-4-methoxyphenol (BHA) cedido pelo fabricante Eastman Chemicals (USA). Os fluídos de base de óleo moringa epoxidados foram aditivados conforme pode ser visto na Tabela 1.

A formulação dos pacotes de aditivos (Tabela 2) foram feitas a partir do planejamento fatorial $2^{3}$ com composto central (Barros Neto et al., 2002), onde foram fixados o antiespumante $(0,30 \mathrm{~g})$ e desemulsificante $(0,15 \mathrm{~g})$ e $5 \%$ de óleo de soja para facilitar a solubilização dos aditivos evitando a precipitação dos mesmos, e variou-se as quantidades do IC, EP e BHA.

Tabela 1 - Fatores e níveis do planejamento fatorial $2^{3}$

\begin{tabular}{c|c|c|c}
\hline Fatores & Símbolos & Nível mínimo & Nível máximo \\
\hline $\begin{array}{c}\text { Inibidor de } \\
\text { Corrosão }\end{array}$ & IC & $0,5 \%$ & $1,5 \%$ \\
\hline Extrema Pressão & EP & $1 \%$ & $5 \%$ \\
\hline Antioxidante & BHA & $0,10 \%$ & $0,30 \%$ \\
\hline
\end{tabular}

Tabela 2 - Matriz de Planejamento $2^{3}$ das formulações dos pacotes de aditivos

\begin{tabular}{c|c|c|c}
\hline FORMULAÇÃO & $\mathrm{A}(\mathrm{mL})$ & $\mathrm{B}(\mathrm{g})$ & $\mathrm{C}(\mathrm{g})$ \\
\hline 1 & 0,67 & 1,30 & 0,13 \\
\hline 2 & 2,07 & 1,30 & 0,13 \\
\hline 3 & 0,67 & 6,50 & 0,13 \\
\hline 4 & 2,07 & 6,50 & 0,13 \\
\hline 5 & 0,67 & 1,30 & 0,39 \\
\hline 6 & 2,07 & 1,30 & 0,39 \\
\hline 7 & 0,67 & 6,50 & 0,39 \\
\hline 8 & 2,07 & 6,50 & 0,39 \\
\hline Pc1 & 1,38 & 3,90 & 0,26 \\
\hline Pc2 & 1,38 & 3,90 & 0,26 \\
\hline Pc3 & 1,38 & 3,90 & 0,26 \\
\hline
\end{tabular}




\section{RESULTADOS E DISCUSSÃO}

Analisando os resultados apresentados na tabela 3 é possível verificar a matriz resposta do planejamento fatorial $2^{3}$ das formulações. As respostas encontradas nesse planejamento foram avaliadas no software Statística 7.0.

Tabela 3 - Matriz resposta do planejamento fatorial $2^{3}$ para o óleo de moringa epoxidado

\begin{tabular}{c|c|c|c|c|c}
\hline Formulação & $\begin{array}{c}\text { Viscosidade } \\
(\mathrm{cSt})\end{array}$ & $\begin{array}{c}\text { I.A.T } \\
(\mathrm{mg} \mathrm{KOH} / \mathrm{g})\end{array}$ & $\begin{array}{c}\text { Corrosão } \\
\text { ao cobre }\end{array}$ & $\begin{array}{c}\text { Estabilidade } \\
\text { Oxidativa }(\mathrm{min})\end{array}$ & $\begin{array}{c}\text { Ponto de Fulgor } \\
\left({ }^{\circ} \mathrm{C}\right)\end{array}$ \\
\hline 1 & 84,03998 & 0,2 & $1 \mathrm{~A}$ & 141,70 & 234 \\
\hline 2 & 82,24678 & 0,27 & $1 \mathrm{~A}$ & 228,82 & 198 \\
\hline 3 & 80,98189 & 0,165 & $1 \mathrm{~A}$ & 215,93 & 204 \\
\hline 4 & 82,47267 & 0,17 & $1 \mathrm{~A}$ & 223,89 & 168 \\
\hline 5 & 77,23929 & 0,135 & $1 \mathrm{~A}$ & 207,95 & 196 \\
\hline 6 & 79,2773 & 0,215 & $1 \mathrm{~A}$ & 183,48 & 168 \\
\hline 7 & 85,22206 & 0,185 & $1 \mathrm{~A}$ & 215,80 & 200 \\
\hline 8 & 86,12337 & 0,27 & $1 \mathrm{~A}$ & 212,79 & 200 \\
\hline PC1 & 86,1800 & 0,22 & $1 \mathrm{~A}$ & 226,38 & 202 \\
\hline PC2 & 85,23402 & 0,2 & $1 \mathrm{~A}$ & 225,30 & 204 \\
\hline
\end{tabular}

Analisando a tabela 3 observa-se que todas as formulações apresentaram melhora para a corrosão ao cobre estando dentro dos padrões exigidos pela ANP para atuar como lubrificante. Algumas formulações diminuíram a acidez total do óleo quando comparada ao epóxido sem aditivos, aperfeiçoando suas qualidades nesse aspecto, e deixando-o dentro das normas exigidas. Em relação à estabilidade oxidativa, o uso de aditivos no epóxido apresentou bons resultados quando comparados ao epóxido puro, obtendo uma estabilidade à oxidação em torno de nove vezes maior.

Na tabela 4 estão presentes os efeitos com o coeficiente de determinação e o " $p$ value" de cada efeito para as variáveis respostas estudadas. Os valores de $p$ destacados na tabela representam a significância estatística dos parâmetros para o intervalo de confiança de $90 \%$ de confiança $(p<0,1)$.

Analisando a Tabela 4 é possível concluir que apenas o aditivo de Extrema Pressão (EP) é significativo para a viscosidade e também a sua interação com o antioxidante BHA. No caso da acidez o Inibidor de Corrosão (IC) é o aditivo é o mais significativo, do mesmo modo que as interações entre IC com o BHA e EP com o BHA. Para a estabilidade oxidativa todos os aditivos e suas interações são significativas. Para o ponto de fulgor somente a interação entre o IC e o BHA não é significativo. 
Tabela 4 - Efeitos com o coeficiente de determinação e o " $p$ value" de cada efeito.

\begin{tabular}{|c|c|c|c|c|c|}
\hline Variável & Parâmetros & Efeitos & Desvio padrão & $p$ & $\mathrm{R}^{2}$ \\
\hline Viscosidade & $\begin{array}{c}\text { Média } \\
\text { IC } \\
\text { EP } \\
\text { BHA } \\
\text { IC x EP } \\
\text { IC x BHA } \\
\text { EP x BHA } \\
\text { IC x EP x BHA }\end{array}$ & $\begin{array}{c}83,28 \\
0,69 \\
3,00 \\
-0,47 \\
0,54 \\
0,81 \\
4,42 \\
-1,11\end{array}$ & $\begin{array}{l}0,2858 \\
0,3352 \\
0,3352 \\
0,3352 \\
0,3352 \\
0,3352 \\
0,3352 \\
0,3352\end{array}$ & $\begin{array}{l}0,00001 \\
0,41163 \\
0,04649 \\
0,55594 \\
0,50724 \\
0,35019 \\
0,02228 \\
0,24098\end{array}$ & 0,63 \\
\hline Acidez & $\begin{array}{c}\text { Média } \\
\text { IC } \\
\text { EP } \\
\text { BHA } \\
\text { IC x EP } \\
\text { IC x BHA } \\
\text { EP x BHA } \\
\text { IC x EP x BHA }\end{array}$ & $\begin{array}{c}0,20 \\
0,06 \\
-0,01 \\
0,00 \\
-0,02 \\
0,02 \\
0,06 \\
0,02 \\
\end{array}$ & $\begin{array}{l}0,0030 \\
0,0035 \\
0,0035 \\
0,0035 \\
0,0035 \\
0,0035 \\
0,0035 \\
0,0035\end{array}$ & $\begin{array}{l}0,0002 \\
0,0136 \\
0,4000 \\
1,0000 \\
0,1679 \\
0,0862 \\
0,0136 \\
0,1318\end{array}$ & 0,98 \\
\hline Estabilidade Oxidativa & $\begin{array}{c}\text { Média } \\
\text { IC } \\
\text { EP } \\
\text { BHA } \\
\text { IC x EP } \\
\text { IC x BHA } \\
\text { EP x BHA } \\
\text { IC x EP x BHA }\end{array}$ & $\begin{array}{c}209,76 \\
17,07 \\
26,62 \\
2,42 \\
-14,42 \\
-30,64 \\
-8,04 \\
25,16\end{array}$ & $\begin{array}{l}0,1645 \\
0,1929 \\
0,1929 \\
0,1929 \\
0,1929 \\
0,1929 \\
0,1929 \\
0,1929\end{array}$ & $\begin{array}{l}0,000001 \\
0,000511 \\
0,000210 \\
0,024512 \\
0,000715 \\
0,000159 \\
0,002296 \\
0,000235\end{array}$ & 0,84 \\
\hline Ponto de Fulgor & $\begin{array}{c}\text { Média } \\
\text { IC } \\
\text { EP } \\
\text { BHA } \\
\text { IC x EP } \\
\text { IC x BHA } \\
\text { EP x BHA } \\
\text { IC x EP x BHA }\end{array}$ & $\begin{array}{c}193,52 \\
-36,41 \\
-17,50 \\
-21,50 \\
-4,50 \\
-0,50 \\
12,50 \\
-4,50\end{array}$ & \begin{tabular}{l|l|}
0,6030 \\
0,7071 \\
0,7071 \\
0,7071 \\
0,7071 \\
0,7071 \\
0,7071 \\
0,7071
\end{tabular} & $\begin{array}{l}0,000010 \\
0,001505 \\
0,006467 \\
0,004299 \\
0,086188 \\
0,757464 \\
0,012559 \\
0,086188\end{array}$ & 0,93 \\
\hline
\end{tabular}

Através a utilização do software Statística 7.0 foi possível obter os modelos matemáticos lineares utilizados para prever os valores de viscosidade, índice de acidez, ponto de fulgor e estabilidade oxidativa, sem necessidade de mais experimentos. Os modelos matemáticos obtidos estão representados nas equações $1,2,3$ e 4 , e apresentam um $\mathrm{R}^{2}$ médio superior a $85 \%$ demonstrando um bom ajuste dos modelos. Esse ajuste pode ser comprovado nos baixos valores do Erro puro para todas as variáveis respostas investigadas, sendo para Viscosidade igual a 0,0121333, para a Acidez 0,0001, para a Estabilidade Oxidativa igual 0,29, e para o Ponto de Fulgor igual a 0,00001.

$\mathrm{Y}_{[\text {Viscosidade }]}=93,13( \pm 0,2858)-3,56 *[\mathrm{IC}]( \pm 0,3351)-2,17 *[\mathrm{EP}]( \pm 0,3351)-$

$46,13 *[\mathrm{BHA}]( \pm 0,3351)+0,74 *[\mathrm{IC} * \mathrm{EP}]( \pm 0,3351)+13,37 *[\mathrm{IC} * \mathrm{BHA}]( \pm 0,3351)+$ $9,78 *[\mathrm{EP} * \mathrm{BHA}]( \pm 0,3351)-2,30 *[\mathrm{IC} * \mathrm{EP} * \mathrm{BHA}]( \pm 0,3351)$ 
$\mathrm{Y}_{[\text {Acidez }]}=0,21( \pm 0,003)+0,063 *[\mathrm{IC}]( \pm 0,003)-0,005 *[\mathrm{EP}]( \pm 0,003)-0,31 *[\mathrm{BHA}]( \pm 0,003)-$ $0,013 *[\mathrm{IC} * \mathrm{EP}]( \pm 0,003)-0,02 *[\mathrm{IC} * \mathrm{BHA}]( \pm 0,003)+0,037 *[\mathrm{EP} * \mathrm{BHA}]( \pm 0,003)+$ $0,036 *[\mathrm{IC} * \mathrm{EP} * \mathrm{BHA}]( \pm 0,003)$

$\mathrm{Y}[$ Estabilidade oxidativa $]=1,10( \pm 0,1645)+123,56 *[\mathrm{IC}]( \pm 0,1929)+32,93 *[\mathrm{EP}]( \pm 0,1929)+$ $577,94 *[\mathrm{BHA}]( \pm 0,1929)-17,53 *[\mathrm{IC} * \mathrm{EP}]( \pm 0,1929)-370,39 *[\mathrm{IC} * \mathrm{BHA}]( \pm 0,01929)-$ $85,79 *[\mathrm{EP} * \mathrm{BHA}]( \pm 0,1929)+52,41 *[\mathrm{IC} * \mathrm{EP} * \mathrm{BHA}]( \pm 0,1929)$

$\mathrm{Y}[$ Ponto de Fulgor $]=288,76( \pm 0,001)-29,69 *[\mathrm{IC}]( \pm 0,001)-9,89 *[\mathrm{EP}]( \pm 0,001)-202,55 *[\mathrm{BHA}]( \pm$ $0,001)+1,22 *[\mathrm{IC} * \mathrm{EP}]( \pm 0,003)+33,86 *[\mathrm{IC} * \mathrm{BHA}]( \pm 0,001)+31,71 *[\mathrm{EP} * \mathrm{BHA}]( \pm 0,001)-$

$9,38 *[\mathrm{IC} * \mathrm{EP} * \mathrm{BHA}]( \pm 0,001)$
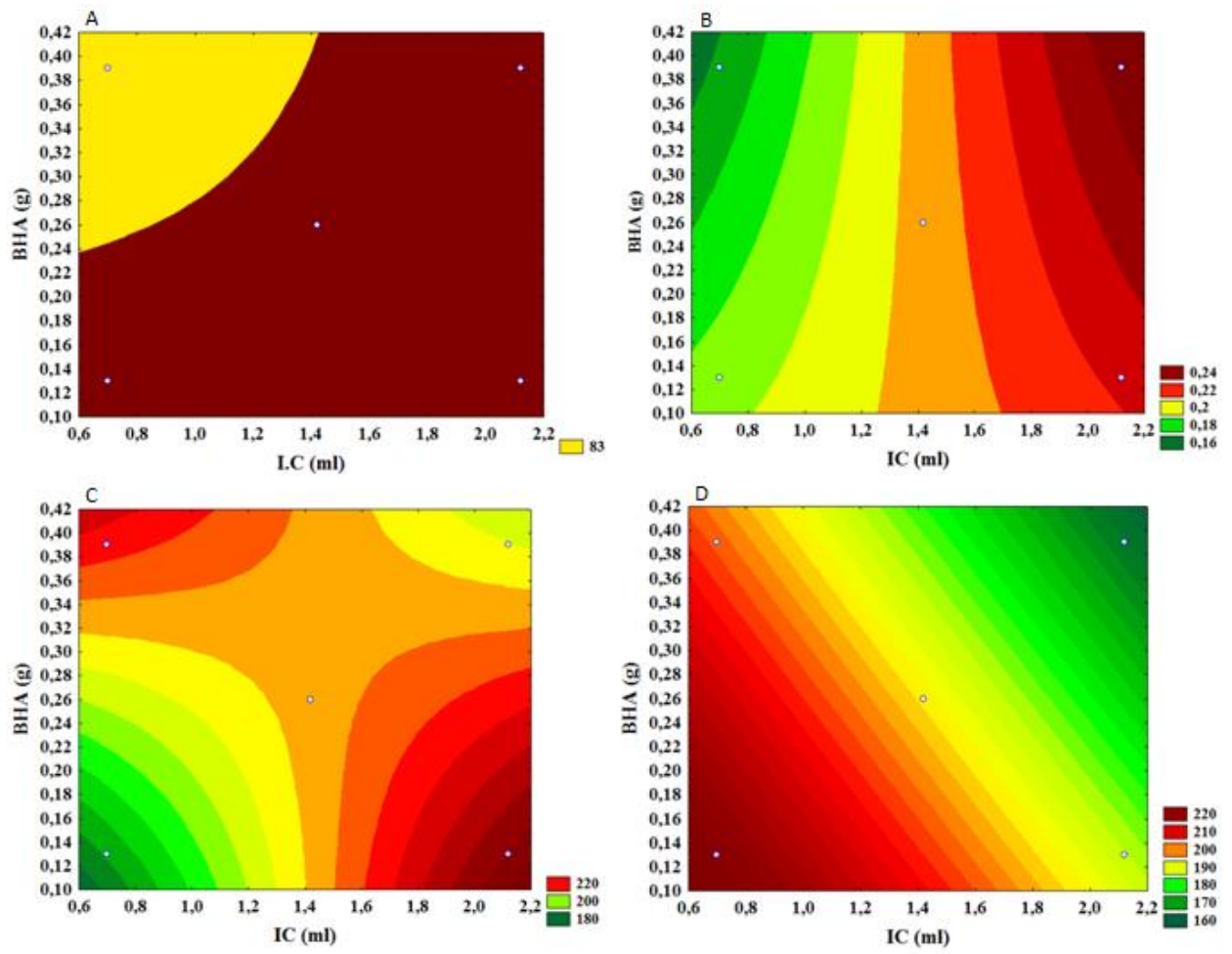

Figura 1 - a) Curva de nível para viscosidade; b) Curva de nível para o Índice de Acidez; c) Curva de nível para Estabilidade oxidativa; d) Curva de nível para o Ponto de Fulgor.

Através do modelo estatístico criado foi possível verificar quais as regiões mais favoráveis para melhorar a aditivação do epóxido. Através da análise das curvas de nível do planejamento fatorial (Figura 1) concluiu-se que para o melhor desempenho do fluido como lubrificante hidráulico é necessário 0,69 mL de IC, 3,9 g de EP e 0, 39 g de BHA obtendo assim resultados de desempenho superior, isso pode ser comprovado experimental obtendo o resultado do índice de acidez total igual a $0,13 \mathrm{mg} \mathrm{KOH} / \mathrm{g}, 211^{\circ} \mathrm{C}$ de ponto de fulgor, $5 \mathrm{~h} 13 \mathrm{~min}$ de estabilidade oxidativa, $83,95 \mathrm{~mm}^{2} / \mathrm{s}$ de viscosidade e $1 \mathrm{~A}$ para o teste de corrosão ao cobre. $\mathrm{Na}$ tabela 5 expõe a caracterização do óleo de moringa epoxidado antes e depois da aditivação, confirmando que a utilização de aditivos melhora as característica do fluido lubrificante. 
Tabela 5- Características do óleo de moringa epoxidado antes e depois da aditivação otimizada.

\begin{tabular}{c|cc}
\hline Parâmetro & \multicolumn{2}{|c}{ Epóxido } \\
& Sem aditivo & Com aditivo \\
\hline Viscosidade a $40^{\circ} \mathrm{C}\left(\mathrm{mm}^{2} / \mathrm{s}\right)$ & $80,37( \pm 0,05)$ & $83,50( \pm 0,06)$ \\
Ponto de Fulgor $\left({ }^{\circ} \mathrm{C}\right)$ & $230( \pm 2)$ & $211,00( \pm 1,41)$ \\
Índice de acidez & $0,20( \pm 0,01)$ & $0,21( \pm 0,02)$ \\
Estabilidade oxidativa $(\mathrm{min})$. & $16,89( \pm 0,6)$ & $204,70( \pm 1,00)$ \\
Corrosão ao cobre & $1 \mathrm{~A}$ & $1 \mathrm{~A}$ \\
Densidade a $40^{\circ} \mathrm{C}\left(\mathrm{g} / \mathrm{cm}^{3}\right)$ & $0,9333( \pm 0,006)$ & $0,9368( \pm 0,025)$ \\
Oxigênio Oxirânico $(\%)$ & $4,08( \pm 0,23)$ & $4,08( \pm 0,23)$ \\
Índice de Iodo, WIJS $\left(\mathrm{mg} \mathrm{de} \mathrm{I}_{2} / \mathrm{g}\right)$ & $6,18( \pm 0,31)$ & $8,18( \pm 0,2)$ \\
\hline
\end{tabular}

Verifica-se na tabela 5 que a viscosidade dos fluídos de moringa aumenta depois da epoxidação e aditivação. O ponto de fulgor dos fluídos cresce com a aditivação e com a epoxidação. Observa-se que após o epóxido ser aditivado ocorre uma queda de quase $10 \%$ do ponto de fulgor do fluído. A acidez do óleo aumenta um pouco após a aditivação e da epoxidação. O índice de iodo diminui e o oxigênio oxirânico aumenta como é esperado em um processo de epoxidação. A corrosão ao cobre não é alterada com e sem a presença de aditivos ou modificação química do óleo. A estabilidade oxidativa do óleo puro aumenta após a aditivação e diminui após a epoxidação, porém observa-se que a aditivação do epóxido proporciona um acréscimo de 12 vezes o valor inicial da estabilidade. Isso comprova que a epoxidação do óleo promove a melhoria das características físicas-químicas dos fluídos lubrificantes de base óleo de Moringa epoxidado.

\section{CONCLUSÕES}

Pode-se concluir que a aditivação melhorou as propriedades físico-químicas do epóxido quando comparado ao epóxido sem aditivos. A análise das curvas de nível geradas pelos modelos matemáticos sugere que para a otimização das formulações é necessário o IC a 1,38 mL, EP a 6,5 g e BHA a 0,13 g para melhor desempenho como biolubrificante, isso pode ser confirmado experimentalmente onde o índice de acidez total foi igual a $0,13 \mathrm{mg} \mathrm{KOH} / \mathrm{g}, 211^{\circ} \mathrm{C}$ de ponto de fulgor, $5 \mathrm{~h} 13 \mathrm{~min}$ de estabilidade oxidativa, $83,95 \mathrm{~mm}^{2} / \mathrm{s}$ de viscosidade e $1 \mathrm{~A}$ para o teste de corrosão ao cobre. Por fim, pode-se confirmar que o biolubrificante desenvolvido a partir do óleo de moringa epoxidado possui grande potencial como lubrificante hidráulico, uma vez que apresentou viscosidade dentro do limite exigido para esse tipo de fluído, com baixa acidez e alta estabilidade oxidativa.

\section{REFERENCIAS}

ABDULKARIM, S.M. ; LONG, K.; LAI, O. M. Frying quality and stability of high-oleic Moringa oleifera seed oil in comparison with other vegetable oils. Food Chem. v. 105, p. 1382-1390, 2007. 
ARBAIN, N. H.; SALIMON, J. Synthesis And Characterization Of Ester Trimethylolpropane Based Jatropha Curcas Oil As Biolubricant Base Stocks. J. Sc.Tech. v. 2, p. 47-58, 2011.

AMERICAN OIL CHEMISTS' SOCIETY (A.O.C.S). Official methods and recommended practices of the American Oil Chemists' Society. 4th ed. Champaign, USA, A.O.C.S., 1995 [A.O.C.S. Recommended Practice Cd 1 - 25]; 1995.

ASTM D 1298. Standard Test Method for Density, Relative Density (Specific Gravity), or API Gravity of Crude Petroleum and Liquid Petroleum Products by Hydrometer. ASTM Inter., 1998.

ASTM D 130. Test Method for Detection of Copper Corrosion from Petroleum Products by the Copper Strip Tarnish Test. ASTM Inter., 2000.

ASTM D 92. Test Method for Flash and Fire Points by Cleveland Open Cup. ASTM Inter.2002.

ASTM D 2983. Test Method for Low-Temperature Viscosity of Lubricants Measured by Brookfield Viscometer. ASTM Inter., 2004.

ASTM D 974. Test Method for Acid and Base Number by Color-Indicator Titration, ASTM Inter., 1997.

BARROS NETO, B.; SCARMINIO, I. S.; BRUNS, R.E. Como Fazer Experimentos. Pesquisa e Desenvolvimento na Ciência e na Indústria. Editora da Unicamp, Campinas, 2002.

EREDA, T. Epoxidação de óleos vegetais, visando à obtenção de lubrificantes industriais. Dissertação (Mestrado em Engenharia Mecânica), Centro Federal de Educação Tecnológica do Paraná, Curso de Pós-Graduação em Engenharia Mecânica e de Materiais, Curitiba, 2004.

ERHAN, S.Z, SHARMA, B.K., LIU, Z., Adhvaryu, A. Lubricant Base Stock Potential of Chemically Modified Vegetal Oils. J. Agric. Food Chem. v.19, p. 8919-8925, 2008.

HWANG, H.-S., ADHVARYU, A. ERHAN, S. Z. Preparation and properties of lubricant basestocks from epoxidized soybean oil and 2 -ethylhexanol. J. Am. Oil. Chem. Soc. v. 80, n. 8, p. 811-815, 2003.

HONARY, L. Biodegradable/Biobased Lubricants and Greases. Mach. Lubr.Mag. Issue Number: 200109, Canadá, 2009.

IUPAC, International Union of Pure and Applied Chemistry. Commission on Oils Fats and Derivatives: Standard Methods for the Analysis of Oils, Fats and Derivatives. London, 1987.

PEREZ, H.I.Q. Produção de biolubrificante para usos especiais. Dissertação (Mestrado em Engenharia Química), Faculdade de Engenharia Química, Universidade Estadual de Campinas, Campinas, 2009.

SALIH, N.; SALIMON, J.; YOUSIF, E.Synthetic biolubricant basestocks based on environmentally friendly raw materials. J. King Saud Univ., Science. v. 24, Issue 3, p. 221$226,2011$. 